\title{
Estudo retrospectivo da obstrução congênita do ducto lácrimonasal
}

\author{
Retrospective study of the congenital \\ lacrimonasal duct obstruction
}

Silvia Helena Tavares Lorena ${ }^{1}$, João Amaro Ferrari Silva²

\section{RESUMO}

Objetivo: O objetivo do projeto de pesquisa será relatar os resultados do tratamento e aspectos epidemiológicos da obstrução congênita do ducto lácrimonasal em crianças. Métodos: Estudo retrospectivo observacional, incluindo 21 crianças portadoras de obstrução congênita do ducto lácrimonasal, atendidas no período de 10 de abril de 2009 a 15 de janeiro de 2010. As crianças foram avaliadas quanto ao sexo, faixa etária, local da obstrução e resultados em relação à sondagem. Resultados: Em relação à epidemiologia da obstrução congênita do ducto lácrimonasal, neste trabalho, podemos afirmar que a distribuição segundo o sexo não mostrou diferença significativa, sendo $42,85 \%$ (9) das crianças do sexo feminino e $57,14 \%$ (12) do sexo masculino. A obstrução ocorreu bilateralmente em $38 \%$, do lado direito em $28,57 \%$ e do lado esquerdo em $33,33 \%$.A $1^{\text {a }}$ sondagem ocorreu antes de 1 ano e 6 meses (Grupo I ): em 7 casos com $100 \%$ de sucesso, entre 2 anos e 3 anos ( Grupo II ): em 10 casos com $80 \%$ de sucesso e acima de 3 anos até 7 anos ( Grupo III ): em 3 casos com $100 \%$ de insucesso . Nenhuma criança necessitou da realização da dacriocistorrinostomia externa, uma vez que a grande maioria melhorou através da sondagem e uma criança através do tratamento conservador. Conclusão: A obstrução congênita do ducto lácrimonasal não tem relação com o sexo.A cura com a $1^{\mathrm{a}}$ sondagem diminui com o avançar da idade, porém há altos índices de cura em crianças acima de 24 meses de vida.Existem outros fatores que corroboram para o insucesso deste procedimento, tais como as anormalidades da cavidade nasal.

Descritores: Obstrução dos ductos lacrimais/congênito; Ducto nasolacrimal; Massagem; Estudos retrospectivos; Crianças.

\footnotetext{
${ }^{1}$ Médica Oftalmologista colaboradora, Pós-graduanda (Doutorado) do Setor de Vias Lacrimais da Universidade Federal de São Paulo - UNIFESP - São Paulo (SP), Brasil;

${ }^{2}$ Chefe do Setor de Vias Lacrimais da Universidade Federal de São Paulo - UNIFESP - São Paulo (SP), Brasil;

Trabalho realizado no Setor de Vias Lacrimais da Universidade Federal de São Paulo - UNIFESP - São Paulo (SP), Brasil.

Recebido para publicação em: 28/7/2010 - Aceito para publicação em 4/2/2011
} 


\section{INTRODUÇÃO}

A obstrução congênita do ducto lácrimonasal é a anormalidade mais frequente da via lacrimal da criança ${ }^{(1)}$. A causa mais comum é a persistência de uma membrana mucosa ao nível da válvula de Hasner e em torno de $96 \%$ se resolve espontaneamente antes do primeiro ano de vida ${ }^{(2)}$.

Alguns trabalhos afirmam que $52 \%$ das crianças no primeiro mês de vida apresentam obstrução congênita do ducto lácrimonasal uni ou bilateral ${ }^{(2)} \mathrm{e}$ ocorre em ambos os sexos de forma semelhante ${ }^{(2)}$.

A produção das lágrimas se inicia por volta dos primeiros 15 dias de vida, sendo assim, as obstruções congênitas da via lacrimal excretora não são diagnosticadas precocemente ${ }^{(2)}$.

A obstrução congênita do ducto lácrimonasal cursa com epífora na presença de olho calmo, com ou sem secreção nos fundos-de-saco conjuntivais. A secreção pode surgir após a expressão na região do saco lacrimal, denotando uma dilatação do saco lacrimal ${ }^{(3)}$.

As obstruções do ducto nasolacrimal podem ser congênitas, adquiridas ou idiopáticas. ${ }^{(3)}$

O diagnóstico diferencial de outras causas de lacrimejamento inclui: glaucoma congênito, conjuntivite neonatal, doenças oculares externas (ceratite, meibomite, blefarite, etc), patologias alérgicas(conjuntivites, rinites,etc), anomalias das pálpebras(entrópio, epibléfaro, distiquíase, etc), hemangioma,meningocele e malformações congênitas ${ }^{(3)}$.

As malformações congênitas do sistema lacrimal de drenagem são mais graves quanto maior for a sua precocidade em relação à época do seu aparecimento ${ }^{(4)}$.

As deformações mais graves são representadas pela ausência ou agenesia total das vias lacrimais de drenagem e também os colobomas de canto medial, que surgem devido à falha de fusão dos processos nasal e maxilar nos primeiros estágios do desenvolvimento que formam as fendas fetais ${ }^{(5)}$.

A imperfuração do ponto lacrimal representa uma falha tardia (6-7meses) no processo de canalização e observa-se a papila com o orifício coberto por fina membrana que pode ser perfurada com a ponta de um dilatador de ponto lacrimal.

A agenesia dos pontos lacrimais e dos canalículos ocorre entre o $4^{\circ}$ e o $6^{\circ}$ mês de gestação e deve ser tratada com a formação de uma intercomunicação direta entre o lago lacrimal e a cavidade nasal (conjuntivorrinostomia) ${ }^{(6)}$.

A blefarofimose é uma síndrome caracterizada por blefaroptose, epicanto, telecanto, e diminuição da fenda palpebral, aparecendo frequentemente uma epífora constante, devido ao bloqueio funcional ao longo dos canalículos.

O telecanto ao afastar os cantos mediais entre si, traciona os canalículos lateralmente, alongando e estreitando o lúmen, dificultando o fluxo lacrimal ${ }^{(7)}$.

A literatura ${ }^{(7)}$ descreve oito tipos de obstrução congênita que podem ocorrer na porção inferior do ducto, sendo citadas principalmente aquelas que terminam no soalho da fossa nasal em um fundo cego e as que possuem desenvolvimento ósseo incompleto.

A mucocele congênita do saco lacrimal é causada por uma prega de mucosa que obstrui o óstio do canalículo comum, ocasionando um abaulamento do saco lacrimal, sem sinais inflamatórios ${ }^{(8)}$.

A fístula do saco lacrimal se forma devido a uma falha na fusão dos processos nasal e maxilar, deixando exteriorizadas algumas células da parte superior da coluna ectodérmica. Algumas células não estando completamente sepultadas podem dar origem a um canalículo supranumerário ou uma fístula, uma vez que são histologicamente semelhantes ${ }^{(9)}$.

As fístulas fazem a comunicação entre a cavidade do saco lacrimal e a superfície da pele, abrindo um orifício abaixo do ligamento medial palpebral. Quando a fístula coexiste com a via lacrimal de drenagem normal, geralmente se fecham espontaneamente, porém quando há obstrução no ducto, a fístula drena o conteúdo do saco lacrimal, causando irritação cutânea.

O diagnóstico da obstrução congênita do ducto nasolacrimal é feito pelo exame clínico (Teste de Milder $^{(10)}$ e TOFO) e pela história clínica ${ }^{(11)}$ de epífora e às vezes, conjuntivite de repetição. A dacriocistografia é um exame complementar que mostra o local da obstrução, porém é pouco utilizada, pois é realizada sob anestesia geral.

A conduta terapêutica consiste na massagem de Criegler em crianças de até um ano de idade e deve ser feita pelo oftalmologista, durante as consultas periódicas $^{(12)}$.

A partir de 1 ano de idade, devemos discutir com os pais a desobstrução instrumental (sondagem das vias lacrimais), uma vez que o seu êxito diminui com o passar dos meses, além de 33\% dos casos evoluírem para a dacriocistite aguda. As sondagens, por vezes, deverão ser repetidas e caso não haja sucesso deveremos proceder à intubação com tubo de silicone ${ }^{(13)}$.

Segundo a literatura, devemos realizar a sondagem em crianças acima de 2 anos de idade, uma vez que a literatura aponta alto índice de cura em crianças acima de 24 meses $^{(14-17)}$ 
A literatura relata outros fatores relacionados com o insucesso da sondagem, além da idade, e entre eles devemos mencionar as anormalidades da cavidade nasal e saco lacrimal dilatado ${ }^{(18)}$.

A dacriocistorrinostomia é indicada nos casos de insucesso da intubação do sistema excretor lacrimal, por volta dos 7 anos de idade ${ }^{(19)}$.

O presente estudo teve por objetivo avaliar os aspectos epidemiológicos da obstrução congênita do ducto lácrimonasal, assim como avaliar o índice de cura e as causas de insucesso com a sondagem terapêutica.

\section{Métodos}

Realizou-se um estudo retrospectivo, utilizando dados dos prontuários de 21 crianças com obstrução congênita do ducto lácrimonasal, atendidas no período de 10 de abril de 2009 a 15 de janeiro de 2010.

As crianças, no período em estudo, foram submetidas à avaliação oftalmológica: inspeção ocular, Teste de Milder e TOFO, inicialmente entre 1 a 3 meses de idade e seguidas em intervalos de 2 meses até a cura da epífora. É mister salientar que as crianças incluídas neste estudo foram examinadas, diagnosticadas e sondadas pela autora deste trabalho.

A inspeção ocular foi realizada com a finalidade de diagnosticar as más posições palpebrais (ectrópio,entrópio) e a existência de massa no canto medial ocular,verificando as suas características (mole,dura,retrátil,pulsátil e a presença de refluxo de secreção para a conjuntiva).

O teste do desaparecimento da fluoresceína (teste de Milder) consistiu na instilação de uma gota de fluoresceína a $2 \%$ no fundo de saco conjuntival do olho suspeito, e o menisco lacrimal foi examinado com um filtro de cobalto da lâmpada de fenda após cinco minutos.A retenção do corante variou de 0 a $4+$ (meniscos de 0 a $1+$ são considerados normais; menisco de $2+$ indica início de obstrução anatômica ou funcional; meniscos de 3 +a 4+ indicam alteração do fluxo lacrimal).

O teste de observação da fluoresceína na orofaringe (TOFO) consistiu na observação da fluoresceína no interior da orofaringe após 60 minutos à instilação no saco conjuntival do olho suspeito, sendo a orofaringe iluminada com uma luz de raios de azul-cobalto.Quando se notou a fluorescência na orofaringe o teste foi considerado positivo, significando via lacrimal pérvia.

Os critérios de inclusão foram:

- crianças na faixa etária de 1 mês a 7 anos;

- crianças com sintoma de epífora desde o nascimento,com ou sem secreção conjuntival.
Os critérios de exclusão foram:

- crianças na faixa etária abaixo de 1 mês e acima de 7 anos.

- criança com lacrimejamento reflexo.

- criança com má posição pálpebral (entrópio, ectrópio, paralisia facial);

- criança com diminuição do tônus do músculo orbicular,ocasionando insuficiência da bomba lacrimal (incluindo também criança com a Síndrome de Down).

Considerando as variáveis desse estudo: tratamento, idade, sexo, cor, número de sondagens realizadas e resultados serão analisadas através do teste de prevalência das frequências.

\section{Resultados}

Em relação à epidemiologia da obstrução congênita do ducto lácrimonasal, podemos afirmar que a distribuição segundo o sexo não mostrou diferença significativa, sendo $42,85 \%$ (9) das crianças do sexo feminino e $57,14 \%$ (12) do sexo masculino, conforme ilustra a Figura 1.

A obstrução ocorreu bilateralmente em $38 \%$, do lado direito em $28,57 \%$ e do lado esquerdo em $33,33 \%$, conforme ilustra a Figura 2.

A secreção mucopurulenta foi diagnosticada em $20 \%$ (2) das crianças entre 2 anos e 3 anos ( Grupo II ) e em 100\% (3) crianças acima de 3 anos até 7 anos (Grupo III ), denotando dilatação do saco lacrimal através do teste do refluxo, conforme ilustra a Figura 3. É importante salientar que estas crianças tiveram insucesso na $1^{\mathrm{a}}$ sondagem, necessitando de uma $3^{\text {a }}$ para a resolução do quadro de epífora e secreção.

A $1^{\text {a }}$ sondagem ocorreu antes de 1 ano e 6 meses (Grupo I): em 7 casos com $100 \%$ de sucesso, entre 2 anos e 3 anos (Grupo I ): em 10 casos com 50\% de sucesso e acima de 3 anos até 7 anos (Grupo III): em 3 casos com $100 \%$ de insucesso necessitando duas novas sondagens para surtir bom resultado, conforme ilustra a Figura 4.

O sexo não é fator prognóstico, uma vez que a cura com a sondagem ocorreu igualmente em ambos os sexos. Desta forma, 9 crianças do sexo feminino e 11 do sexo masculino mellhoraram com sondagem e uma criança de sexo masculino melhorou com a massagem de Criegler.

Nenhuma criança necessitou da realização da dacriocistorrinostomia externa e apenas uma criança com 8 meses apresentou cura com o tratamento conservador. 


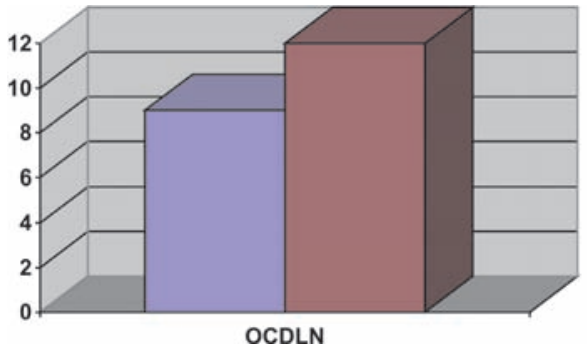

\section{DFem
$\square$ Mas}

Figura 1: Relação do sexo com a obstrução congênita do ducto lácrimonasal (OCDLN)

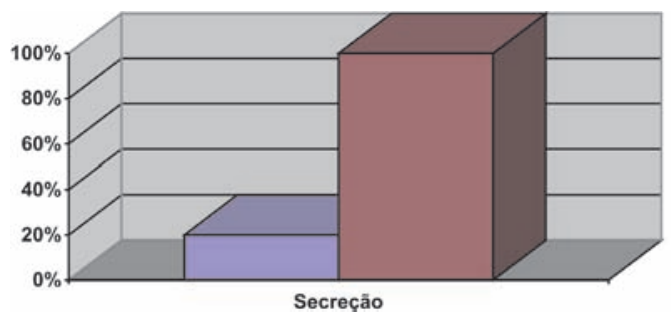

Figura 3: Distribuição porcentual da presença de secreção mucopurulenta entre o Grupo I e Grupo II

\section{Discussão}

A canalização do ducto lácrimonasal pode se completar logo após o nascimento ou mais tarde, podendo ocorrer cura espontânea até 1 ano de vida em cerca de $80 \%$ a $90 \%$ dos $\operatorname{casos}^{(2)}$, fato que ocorreu em uma criança com idade de 8 meses.

Neste trabalho, constatamos que a incidência da obstrução congênita do ducto lácrimonasal ocorreu de forma semelhante em ambos os sexos, o que corrobora com os dados da literatura ${ }^{(3)}$.

Para o diagnóstico da obstrução congênita da via lacrimal excretora foram realizados os testes de Milder (3) e de TOFO, sendo que a dacriocistografia não foi realizada, uma vez que este exame é realizado sob anestesia geral.

A cura com a $1^{a}$ sondagem ocorreu em $100 \%$ em crianças do Grupo I, em $80 \%$ em crianças do Grupo II e em nenhuma criança do Grupo III, tais resultados nos faz concluir que há correlação entre o avanço da idade e a diminuição do índice de cura ${ }^{(14)}$.

Os resultados obtidos nos permite concluir que devemos realizar a sondagem em crianças acima de 2 anos de idade, uma vez que a literatura aponta alto índi-

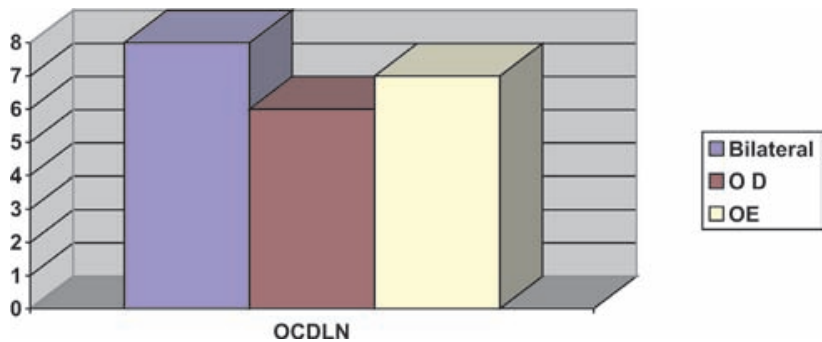

Figura 2: Relação da lateralidade com a obstrução congênita do ducto lácrimonasal (OCDLN)

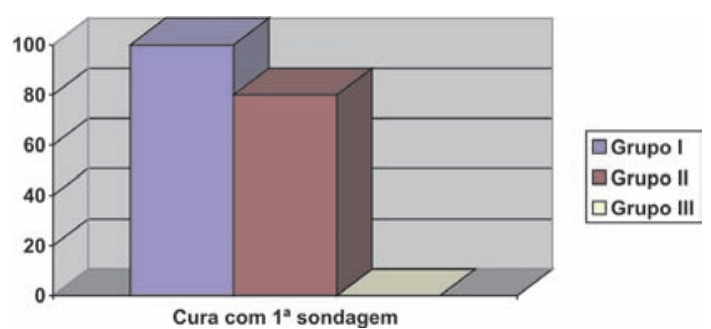

Figura 4: Relação de cura da OCDLN com a $1^{\mathrm{a}}$ sondagem nos diferentes grupos etários

ce de cura em crianças acima de 24 meses $^{(17)}$.

Existem outros fatores relacionados com o insucesso da sondagem, além da idade e entre elas devemos mencionar as anormalidades da cavidade nasal e saco lacrimal dilatado ${ }^{(18)}$.

O sexo não é fator prognóstico, uma vez que a cura com a sondagem ocorreu igualmente em ambos os sexos.

Quando não há resultados satisfatórios com a sondagem, a dacriocistorrinostomia externa deve ser realizada em crianças a partir de 7 anos de idade ${ }^{(19)}$, o que não ocorreu com as crianças desse estudo, uma vez que a epífora se resolveu mediante sondagem das vias lacrimais e uma criança obteve sucesso com o tratamento conservador.

\section{Conclusão}

A obstrução congênita do ducto lacrimonasal não tem relação com o sexo. A cura com a $1^{a}$ sondagem diminui com o avançar da idade, porém há altos índices de cura em crianças acima de 24 meses de vida. Outros fatores corroboram para o insucesso deste procedimento, tais como as anormalidades da cavidade nasal. 


\begin{abstract}
Purpose: The goal of this research is to relate the results of congenital lacrimonasal duct obstruction treatment and epidemic aspects in children. Methods: This is a retrospective study of 21 children with congenital lacrimonasal duct obstruction attended in the period of 9 months approximately (april 2009 to january 2010). The children were evaluated regarding the sex, age, and side of obstruction (right, left or bilateral). Results: Nine of the children were female and 12 were male $(42.85 \%$ versus $57.14 \%$. Therefore there was no significant statistically difference related to gender. The obstruction occurred bilaterally in $38 \%$ of the cases, on the right in $28,57 \%$ and on the left in 33,33\%. Seven children were operated on for the first time before 1 and a half year old ( $1^{\text {st }}$ Group) with a $100 \%$ of success. In 10 children the surgery were performed between 2 an 3 years old ( $2^{\text {nd }}$ Group) with $80 \%$ of success and on the $3^{\text {rd }}$ group there was 3 children (above 3 and till 7 years old) that were treated surgically with $100 \%$ of unsuccess. Conclusion: The congenital lacrimonasal duct obstruction were not related to the gender of the children. The treatment were very succefull during the 1st year of age.

Keywords: Lacrimal duct obstruction/congenital; Nasolacrimal duct; Massage; Retrospective studies; Children
\end{abstract}

\section{RefERÊNCIAS}

1. Guerry D erd, Kendig EL Jr. Congenital impatency of the nasolacrimal duct.Arch Ophthalmol. 1948;39(2):193-204.

2. Schellini SA, Hoyama E, Rocha MC, Rugollo A Jr, Padovani CR. Evaluation of lacrimal system outflow in the newborn by dye tests. Orbit. 2000;19(3):137-42.

3. Zappia RJ, Milder B. lacrimal drainage funtion. The fluorescein dye disappearance test. Am J Ophthalmol.1972;74(1):160-2.

4. Mann I: Developmental abnormalities of the eye.2nd ed.London:British Medical Association; 1957. p. 371.

5. Rougier J;Tessier P;Hervouet F; Woillez M; Lekieffre M\& Derome P:Chirurgie plastique orbito-palpébrale. Paris:Masson; 1977. p.191-208.

6. Jones LT: The Cure of epiphora due to canalicular disorders, trauma and surgical failures on the lacrimal passages. Trans . Am. Acad. Ophthalmol. Otolaryngol.1962;66:506-24.

7. França VP;Bongiovanni CS;Bessa H;Caldato R\& Soares EJC:Ectrópio .In:Cirurgia Plástica Ocular, Soares EJC;Moura EM\&Gonçalves JOR, editores Cirurgia pástica ocular. São Paulo: Roca;1997. p.156-9

8. Jones LT, Wobig JL. Congenital anormalites of lacrimal system. In:Jones LT,WobigJL. Surgery of the eyelid and lacrimal system. Birmingham: Aesculaphius; 1976. p.157-73.

9. Pashby R, Hurwitz JJ. Pediatric lacrimal disease. In: Hurwitiz JJ, editor. The lacrimal system. Philadelphia: Lippincott-Raven; 1996.p. 237-41.

10. Flach A. The fluorescein appearance test for lacrimal obstruction. Ann Ophthalmol.1979;11(2):237-42.

11. Jones LT. The lacrimal secretory system and its treatment. Am J Ophthalmol. 1966;62(1):47-60.
12. Da Pozzo S, Pensiero S, Perissutti P. [Management of congenital nasolacrimal duct obstruction. Timing of probing]. Minerva Pediatr. 1995;47(6):209-13.Italian.

13. el-Mansoury J, Calhoun JH, Nelson LB,Harley RD. Results of late probing for congenital nasolacrimal duct obstruction. Ophthalmology. 1986;93(8):1052-4.

14. Robb RM. Success rates of nasolacrimal duct probing at time intervals after 1 year of age. Ophthalmology. 1998;105(7):13079; discussion 1309-10.

15. Yap EY, Yip CC. Outcome of late probing for congenital nasolacrimal duct obstruction in Singapore children. Int Ophthalmol. 1997-1998;21(6):331-4.

16. Zwaan J. Treatment of congenital nasolacrimal duct obstruction before and after the age of 1 year. Ophthalmic Surg Lasers. 1997;28(11):932-6.

17. Chaim MP, Bison SHDF, Silva JAF. Obstrução congênita do ducto lacrimonasal: estudo de 284 pacientes. Arq Bras Oftalmol. 1996;59(5):519-21.

18. Schellini SA, Gaiotto PC, Schellini RC, Silva MRBM. Obstrução nasolacrimal congenital: diagnóstico e tratamento. Ver Bras Oftalmol. 1994;53(5):47-53.

19. Barnes EA, Abou-Rayyah Y, Rose GE. Pediatric dacryocystorhinostomy for nasolacrimal duct obstruction. Ophthalmology. 2001;108(9):1562-4. 